\title{
Flexible ureteroscopy for renal stone without preoperative ureteral stenting shows good prognosis
}

\author{
Jiaqiao Zhang ${ }^{1}$, Chuou Xu ${ }^{2}$, Deng He ${ }^{1}$, Yuchao Lu ${ }^{1}$, Henglong Hu ${ }^{1}$, Baolong Qin ${ }^{1}$, Yufeng Wang ${ }^{1}$, Qing \\ Wang ${ }^{1}$, Cong Li ${ }^{1}$, Shaogang Wang ${ }^{\text {Corresp., }}{ }^{1}$, Jihong Liu ${ }^{1}$ \\ ${ }^{1}$ Department and Institute of Urology, Tongji Hospital, Tongji Medical College, Huazhong University of Science and Technology, Wuhan, People's Republic \\ of China \\ 2 Department of Radiology, Tongji Hospital, Tongji Medical College, Huazhong University of Science and Technology, Wuhan, People's Republic of China \\ Corresponding Author: Shaogang Wang \\ Email address: sgwangtjm@163.com
}

Purpose To clarify the outcome of flexible ureteroscopy for management of renal calculi without preoperative stenting. Methods A total of 171 patients who received 176 flexible ureteroscopy procedures for unilateral renal stones were reviewed. All procedures were divided into two groups depending on whether they received ureteral stenting preoperatively. Baseline characteristics of patients, stone burden, operation time, stone free rates, and complications were compared between both groups. Results Successful primary access to the renal pelvis was achieved in 104 of 114 (91.2\%) patients without preoperative stenting, while all procedures with preoperative stenting $(n=62)$ were successfully performed. A Total of 156 procedures were included for further data analysis (56 procedures in stenting group and 100 in non-stenting group). No significant differences was found regardless of a preoperative stent placement in terms of stone free rate $(73.2 \%$ with stenting vs. $71.0 \%$ without, $P=0.854)$, operative time $(70.4 \pm 32.8$ with stenting vs $70.2 \pm 32.1$ without, $P=0.969)$. Conclusions Flexible ureteroscopy for management of renal stone without preoperative ureteral stenting are associated with well outcome in short term follow-up. Our study may help patients and doctors to decide if an optional stent is placed or not. 
1 Flexible ureteroscopy for renal stone without preoperative ureteral stenting shows good

2 prognosis

3 Jiaqiao Zhang a, Chuou $\mathrm{Xu}{ }^{\mathrm{b}}$, Deng $\mathrm{He}^{\mathrm{a}}$, Yuchao Lu ${ }^{\mathrm{a}}$, Henglong $\mathrm{Hu}{ }^{\mathrm{a}}$, Baolong Qin ${ }^{\text {a }}$, Yufeng

4 Wang ${ }^{\text {a }}$, Qing Wang ${ }^{\mathrm{a}}$, Cong $\mathrm{Li}^{\mathrm{a}}$, Shaogang Wang ${ }^{\mathrm{a}}$, Jihong Liu ${ }^{\mathrm{a}}$

5 Affiliation:

6 a Department and Institute of Urology, Tongji Hospital, Tongji Medical College, Huazhong

7 University of Science and Technology, Wuhan, Hubei, People's Republic of China

8 b Department of Radiology, Tongji Hospital, Tongji Medical College, Huazhong University of

9 Science and Technology, Wuhan ,Hubei, People’s Republic of China

10 Corresponding author:

11 Shaogang Wang, Department and Institute of Urology, Tongji Hospital, Tongji Medical College,

12 Huazhong University of Science and Technology, 1095 Jiefang Ave., Wuhan 430030, Hubei,

13 People's Republic of China

14 Tel: 86-27-83663460

15 E-mail: sgwangtjm@163.com 


\section{Abstract}

17 Purpose To clarify the outcome of flexible ureteroscopy for management of renal calculi without 18 preoperative stenting.

19 Methods A total of 171 patients who received 176 flexible ureteroscopy procedures for unilateral renal stones were reviewed. All procedures were divided into two groups depending on whether

21 they received ureteral stenting preoperatively. Baseline characteristics of patients, stone burden, 22 operation time, stone free rates, and complications were compared between both groups.

23 Results Successful primary access to the renal pelvis was achieved in 104 of 114 (91.2\%)

24 patients without preoperative stenting, while all procedures with preoperative stenting $(\mathrm{n}=62)$

25 were successfully performed. A Total of 156 procedures were included for further data analysis

26 (56 procedures in stenting group and 100 in non-stenting group). No significant differences was

27 found regardless of a preoperative stent placement in terms of stone free rate (73.2\% with

28 stenting vs. $71.0 \%$ without, $P=0.854$ ), operative time (70.4 \pm 32.8 with stenting vs $70.2 \pm 32.1$

29 without, $P=0.969)$.

30 Conclusions Flexible ureteroscopy for management of renal stone without preoperative ureteral

31 stenting are associated with well outcome in short term follow-up. Our study may help patients

32 and doctors to decide if an optional stent is placed or not. 


\section{Introduction}

34 Improvement of the instruments as well as endoscopic technology has made fURS as an

35 increasingly popular treatment option for patients with renal stones [1-3]. Indication for fURS

36 also has been extended, even for stone larger than 2cm [4, 5]. Preoperative stenting is frequently

37 used to allow passive ureteral dilatation, which is supposed to facilitate the passage of a flexible

38 ureteroscope or ureteral access sheath (UAS). In a study, double-J stent inserted 5-10 days before

39 fURS was recommended as a part of standardized technique to achieve superior results for

40 treatment of renal stones [6]. However, a preoperative stenting inevitably leads extra cost and

41 time, as well as complications such as flank pain, sexual dysfunction, bothersome urinary

42 symptoms and potential urinary tract infection [7-9]. Then, patient compliance is an issue for

43 urologists. In addition, urologists have better skill and more confidence to successfully perform

44 fURS procedures for patients without preoperative stenting with increased experience. For the

45 above reasons, more and more fURS procedures without preoperative stenting were carried out in

46 our department.

47 However, the outcome of Flexible Ureteroscopic Treatment for renal stones without preoperative

48 stenting is undefined. Hence, we reviewed a series of cases to seek the answer. 
49

50

51

52

53

54

\section{Material and Methods}

This study was approved by the ethics committee of Tongji Hospital, Tongji Medical College, Huazhong University of Science and Technology. All unilateral fURS procedures from between June 2013 and May 2015 were reviewed. All patients included underwent fURS as initial attempt or substitute for previous failed ESWL or PCNL procedures to treat solitary as well as multiple renal calculi. The patients with the presence of ipsilateral ureteral stones were excluded. All patients were classified into two groups depending on whether they received a preoperative stent. The patients in stenting group received preoperative stenting $(5 \sim 8 \mathrm{~F})$ for persistent renal colic, fever, insufficient renal function, or just to dilate the ureter to facilitate subsequent fURS. The patients in non-stenting group had not received stenting before fURS.

All procedures were performed by experienced urologists in our department according to standard operative protocols. Urinary tract infection was controlled before all operations. For patients with preoperative stenting, firstly the stents was removed through ureteroscopy (URS) or cystoscopy. And for patients without preoperative stenting, a rigid URS was performed to detect the whole ureter at first. Then a guidewire was inserted into ureter and UAS (12/14F, Cook) was placed routinely. A 7.5F flexible ureteroscope (Storz, Germany) with a $200 \mu \mathrm{m}$ laser fibre was used for laser lithotripsy. Nitinol stone retrieval baskets were also used if necessary at surgeon discretion. All patients received ureteral stent at the end of operations. Then all patients underwent KUB or non-contrast CT about 4 weeks and 3 months after treatment, and absence of any stone or residual stone fragment $\leq 4 \mathrm{~mm}$ was considered as stone free. 
69 We retrospectively recorded data from patient documentation, radiologic images (including X-

70 ray, IVU, and computed tomography), reports of anaesthesia and operation. All radiologic images

71 were checked collaboratively by an urologist and a radiologist. Number, location and linear

72 diameter of stones visible in radiologic images were noted in detail. Fever was defined as

73 postoperative body temperature $\geq 38$. The procedures were performed by a total of five urologists.

74 Statistical analysis was performed by Statistical Package for the Social science version13.0.

75 Continuous variables with normal distribution and without normal distribution were performed

76 with Student’s t-test and Mann-Whitney U-test, respectively. Categorical variables were

77 performed with chi-squared test or Fisher's exact test. Potential factors associated with stone free

78 rate including $\mathrm{BMI}\left(\mathrm{kg} / \mathrm{m}^{2}\right)$, stone size $(\mathrm{mm})$, stone number, presence of preoperative stenting,

79 type of anaesthesia and presence of hydronephrosis were analyzed by multivariate logistic

80 regression analysis. A $P$ value of $<0.05$ was considered statistically significant. 


\section{Results}

82 A total of 176 unilateral procedures of fURS from 171 individual patients were included. The

83 figure 1 shows the clinical outcome of all patients. Majority (104 of the 114) of the patients

84 without a preoperative stent were successfully performed fURS, while the left 10 patients

85 underwent a failed attempt for access to the renal pelvis was not achieved. Of the 10 patients, five

86 patients received a stent placement and subsquent fURS, 4 patients underwent a conversion to

87 PCNL procedure,and one patient received a stent followed by ESWL treatment. All of 62 patients

88 with a preoperative doule-J stent (including the above mentioned five patients who received a

89 stentin first failed attempt) were successfully performed fURS (figure 1).

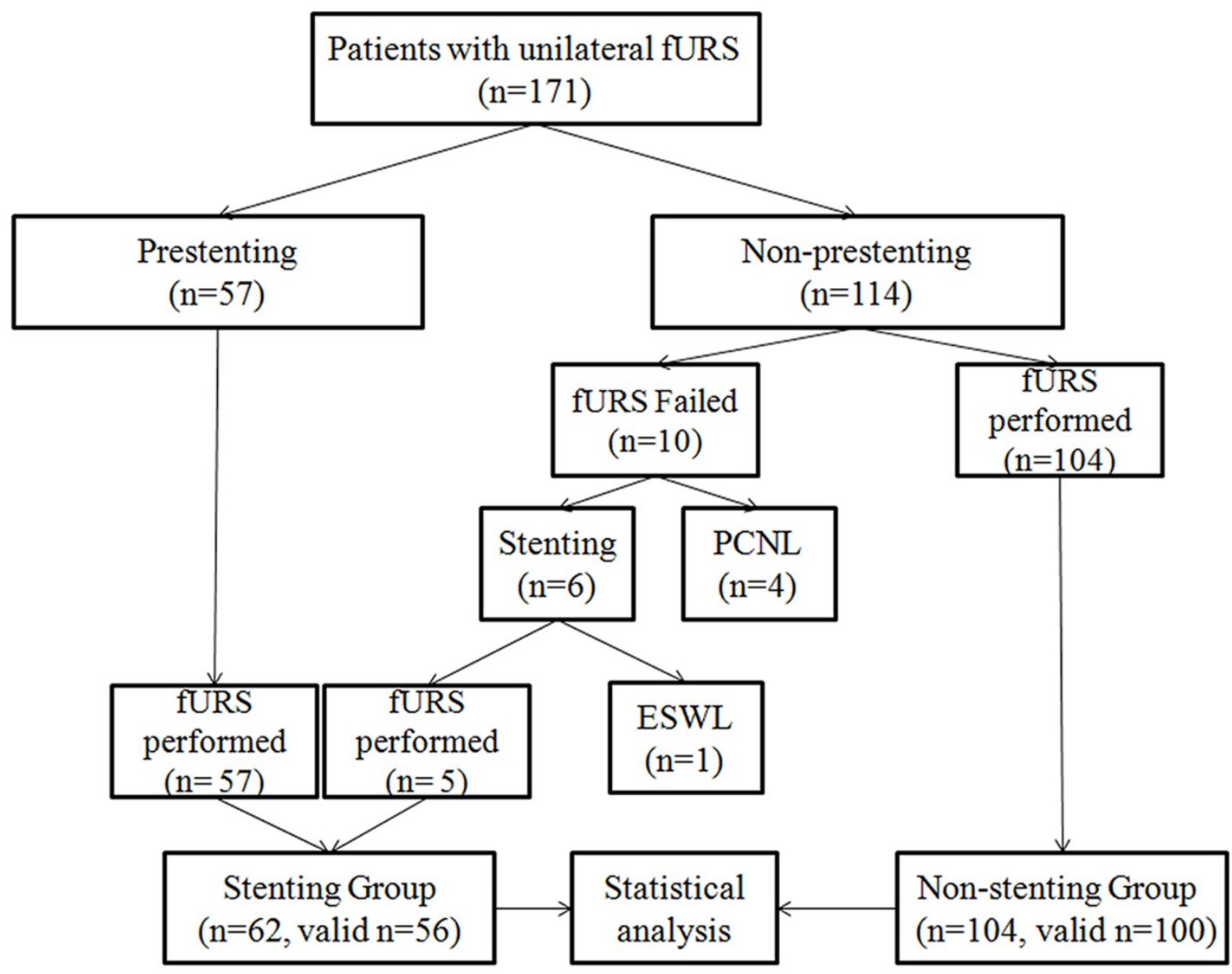

90 Figure 1. Flow diagram and patient disposition.(fURS= flexible ureteroscopy; ESWL= 
91 Extracorporal shock wave lithotripsy; PCNL= Percutaneous nephrolithotomy)

92 As the figure 1 showed, a tota1 of 166 procedures successfully entered into renal pelvis to

93 perform fURS lithotripsy. Of the 166 procedures, ten procedures with incomplete data were not

94 enrolled for further data analysis. There were no statistically significant differences between

95 preoperative stenting group $(n=56)$ and non-stenting group $(n=100)$ in terms of age, gender, side,

96 BMI, presence of hydronephrosis, type of anaesthesia, operation time. Stone number, stone site,

97 and stone burden were also similar in both groups. Positive rate of preoperative urine culture was

98 higher in stenting group. Stone free rate was similar in both groups. Procedures for solitary stone

99 accounted for $71.2 \%$ (111 of 156). For single stone, stone free rate was highest for stone in renal

100 pelvis, and lowest in lower pole. Stone free rates were also similar for patients with solitary stone

101 in both groups (Table 1). Multivariate assessment revealed stone size rather than preoperative

102 stenting was the independent predictor of stone free rate after fURS in this study. (Table 2)

103 Table 1 Patient characteristics in stenting and non-stenting group

\begin{tabular}{lccc}
\hline & Stenting group & Non-stenting group & $P$ \\
\hline Procedures & 56 & 100 & \\
Age (year) & $51.4 \pm 12.8$ & $47.6 \pm 13.2$ & 0.080 \\
Gender (Men/Women) & $32 / 24$ & $62 / 38$ & 0.610 \\
Side (Right/Left) & $24 / 32$ & $47 / 53$ & 0.738 \\
BMI (kg/m ${ }^{2}$ ) & $24.2 \pm 3.6$ & $23.7 \pm 3.1$ & 0.381 \\
Hydronephrosis(+/-) & $20 / 36$ & $39 / 61$ & 0.733 \\
Urine culture(+/-) & $13 / 43$ & $7 / 93$ & 0.006 \\
Anaesthesia & $30 / 26$ & $48 / 52$ & 0.617 \\
(Epidural/General) & & & \\
Operation time (min) & $70.4 \pm 32.8$ & $70.2 \pm 32.1$ & 0.969 \\
Stone size (mm) & $18.1 \pm 8.1$ & $18.4 \pm 7.7$ & 0.812 \\
$\quad<\mathbf{1 0}$ & 7 & 7 & \\
$10-20$ & 29 & 63 &
\end{tabular}


$>20$

Solitary/multiple stone

Solitary stone location

Upper pole

Middle pole

Lower pole

Renal pelvis

Stone free rate

For Solitary stone
20

$40 / 16$

3

5

15

17

$73.2 \%(41 / 56)$

$71.0 \%(71 / 100)$

0.854

0.955

$85.0 \%(34 / 40) \quad 77.5 \%(55 / 71) \quad 0.458$

104 105

Table 2. Result of multiple logostic regression analysis to determine factors associated with stone free rate.

\begin{tabular}{lllll}
\hline Varible & Category & OR & $95 \%$ CI & $p$ value \\
\hline BMI $\left(\mathrm{kg} / \mathrm{m}^{2}\right)$ & & 1.027 & $0.899-1.173$ & 0.699 \\
Stone size $(\mathrm{mm})$ & & 0.407 & $0.206-0.807$ & 0.010 \\
Stone number & $1 \mathrm{vs} \geq 2$ & 0.423 & $0.147-1.216$ & 0.110 \\
Preoperative stenting & Present vs absent & 0.799 & $0.333-1.919$ & 0.616 \\
Anaesthesia & General vs epi & 0.907 & $0.392-2.101$ & 0.820 \\
Hydronephrosis & Absent vs present & 1.070 & $0.448-2.558$ & 0.879 \\
\hline
\end{tabular}

Complication rates were low in both groups except for fever. Surprisingly, up to $17.9 \%$ of patients with preoperative stent experienced fever postoperatively. Patients without preoperative stenting had a much lower incidence of fever compared with that (6\% vs. $17.9 \%, P=0.027)$. Perforations of renal pelvis or ureter were more common for patients without a preoperative stent, and were low in both groups. All perforations were not severe, and treated with a postoperative stent. No complications of ureter avulsion occurred in both groups. Severe complications such as bleeding with transfusion or urosepsis were rare. (Table 3) 


\begin{tabular}{cllcc}
\hline & \multicolumn{3}{c}{$\begin{array}{l}\text { stenting } \\
\text { group }\end{array}$} \\
\hline Fever & (GradeII) & $10(17.9 \%)$ & $6(6 \%)$ & 0.027 \\
$\begin{array}{l}\text { Renal } \\
\text { pelvis /Ureter }\end{array}$ & (Grade IIIb) & $1(1.8 \%)$ & $4(4 \%)$ & \\
perforation & & & \\
Transfusion & $($ Grade II) & $1(1.8 \%)$ & 0 \\
Urosepsis & (Grade IVb) & $1(1.8 \%)$ & $1(1 \%)$ & \\
\hline
\end{tabular}

115 Disscussion

116 As one of common available treatment options, fURS has occupied more and more positions 117 formerly held by PCNL and ESWL. However, limited data have been referred to the role of 118 preoperative stenting in the treatment of renal calculi by fURS [10-14]. Shields et al reviewed a 119 cohort of patients undergoing URS for upper urinary tract calculi and concluded that preoperative 120 stent was positively associated with success, however, without statistical significance [11]. 121 Lumma et al analyzed data of 550 ureterorenoscopies treated for stones in ureter and renal pelvis. 122 Data indicated that patients with preoperative stent had improved results in stone free rate and 123 complication rate apart from distal ureteral stones [10]. Kawahara et al analyzed 51 patients with 124 large $(>15 \mathrm{~mm})$ stone, and concluded that ureteroscopy success rate was higher in the stenting 125 group and may improve SFR [13]. However, most studies were referred to patients with ureteral 126 and renal calculi, and instruments and methods applied for ureteral calculi are quite different 127 from renal calculi. In addition, fURS occasionally will not pass beyond the ureter to reach renal 128 pelvis, which have been mentioned limitedly [15-17]. Therefore, the aim of this study was to 129 systematically clarify the surgical outcome of fURS without preoperative stenting for renal 130 calculi.

131 Of the 114 patients without preoperative stenting, 10 patients underwent a failured procedure for 
132 access to the renal pelvis was not achieved. In these patients, a narrow ureteric lumen or tortuous 133 ureter caused the failure of access, even though no evidence of obstruction in ureter was found 134 preoperatively or intraoperatively. Half of the 10 patients received a ureteral stent for a period of 135 time (7 40d) and underwent a second attempt in which narrow ureteric lumen or tortuous ureter 136 disappeared. Obviously, the preoperative stenting passively dilated the ureter and facilitated access to renal pelvis in them. A study by Ambani et al retrospectively analyzed 41 patients who underwent ureteral stenting after an initial failed attempt of fURS. The second fURS was performed in these patients after 4 to 34 days and succeeded in 38 patients (93\%) [15]. Therefore, when a difficult ureter was faced in first attempt of fURS, placement of a stent and subsequent

second attempt was optional and even encouraged.

In our study, stone free rate (3 months after operation) in patients with preoperative stenting was higher compared to patients without preoperative stenting, however without statistical significance $(73.2 \% \%$ vs. $71.0 \%, P=0.0 .854)$. This result was not consistent with some previously published data $[10,11,18]$. One possible reason was that ureteral stones account for a high proportion of all stones in these studies. Multivariate assessment also revealed that preoperative stent was not the independent predictor of stone free rate after fURS in our study which was similar with a previous study [19]. In our opinion, once the fURS successfully arrived at renal pelvis, stone free rate for renal calculi would be more likely affected by stone characteristics and pelvicalyceal anatomy rather than the existence of preoperative stenting according to our experience and some published data [20-22].

152 Stone free rate was not very high in our study. One reason may be that stone size $(18.4 \pm 7.7 \mathrm{~mm})$ 
153 in our study was higher than many studies [6, 23, 24]. Although non-contrast CT was the most 154 accurate modality for follow-up of urolithiasis, we also performed KUB for follow-up for its 155 advantages of less cost and radiation exposure.

156 The operation time was similar in both groups. However, different results had been reported. 157 Lumma et al found that the operation time in patients with preoperative stent was extended by 4.9 158 min compared to patients without stent, and attributed this to stent extraction [10]. Another study 159 indicated that preoperative stenting decreased operative time of URS for stones (mainly in the 160 ureter). The possible reason may be that preoperative stenting dilated the ureter and allowed a 161 bigger ureteral access sheath and better irrigation [18]. In our experience, it would consume some 162 time to remove preexist stents, however, a procedure of rigid URS was not necessary before 163 placing the UAS in these patients generally.

164 Severe complications were rare in our study, however, postoperative fever rate was much higher compared with other complications. And patients with preoperative stenting had a higher incidence of fever compared with without $(17.9 \%$ vs. $6.0 \%, P=0.027)$. The reason may be that majority of patients with preoperative stenting in this study received a stent just because of existence of fever or urinary tract obstruction and patients in stenting group indeed had a significant higher rate of positive urine culture. So we could not conclude that preoperative stenting increased postoperative fever rate, although an extended time of stenting may cause potential urinary tract infection (UTI) [25]. And the incidence of fever in our patients without 172 preoperative stenting was similar with the results (3.6\% for $10-20 \mathrm{~mm}$ renal stones) of CROES 
173 URS Global Study [20].

174 Perforations of ureter or pelvis were not very common in both groups (1.8\% with stent vs. $4 \%$

175 without). Actually, as many mild injuries may be ignored in records of our operations, it was hard

176 to know accurate incidence of ureter injuries for all patients. We speculated that ureter injuries

177 (especially for mild injuries) were common according to our experience as well as published data

178 [26]. However, severe ureter injuries such as ureter avulsion never happened. In our experience,

179 if unreasonable resistance was perceived when passing through the ureter, it was better to

180 terminate the fURS procedure rather than try with a greater force to avoid severe ureter injury. As

181 many above mentioned substitutes could be selected subsequently, an “adventure” was not

182 necessary.

183 A recent published large scale study included 1622 patients with renal stones who were treated 184 with fURS and concluded that preoperative stent increased stone free rates (79.6\% with stent vs. 185 72.9\% without stent) and decreased intraoperative complications [27]. The results of this large 186 scale study are very valuable, however, we still should note that heterogeneity of reasons for 187 preoperative stenting may affect outcome of fURS for renal stones in different studies. And this 188 large scale study indicated that non-stenting fURS for renal stones may have some disadvantages, 189 however, it still had an acceptable prognosis. In addition, it should be emphasized that non190 stenting procedures would benefit patients for less cost and suffering.

191 Our study has some limitations. First, it is a retrospective study from single center. Second, the 
192 complications mentioned in our study were early stage complications, while late complications 193 such as ureter stricture were not concerned due to limited follow-up time. Despite these facts, our 194 study is one of the largest series to determine the prognosis of non-stenting procedure on 195 management of renal calculi with fURS.

196 Our study showed that majority(91.2\%, 104 of the 114) of the patients without preoperative 197 stenting were successfully performed fURS at first attempt. The procedures of Non-stenting 198 fURS for renal stones have an acceptable prognosis compared with the stenting group in our 199 study. If a preoperative stenting is optional, our study may help the patients and clinicians make a 200 final decision. And large prospective randomized controlled studies are required to further figure 201 out the role of pre-stent, especially for optional cases. 
203

204

205

206

207

208

209

210

211

212

213

214

215

216

217

218

219

220

221

222

223

224

225

226

227

228

229

230

231

232

233

234

235

236

237

238

239

240

241

242
[1] Ghani KR, Wolf JS (2015) What is the stone-free rate following flexible ureteroscopy for kidney stones. Nat Rev Urol 12:281-288

[2] Dauw CA, Simeon L, Alruwaily AF et al (2015) Contemporary practice patterns of flexible ureteroscopy for treating renal stones: Results of a worldwide survey. J Endourol

[3] Wang AJ, Preminger GM (2011) Modern applications of ureteroscopy for intrarenal stone disease. Curr Opin Urol 21:141144

[4] Aboumarzouk OM, Monga M, Kata SG et al (2012) Flexible ureteroscopy and laser lithotripsy for stones $>2 \mathrm{~cm}$ : a systematic review and meta-analysis. J Endourol 26:1257-1263

[5] Breda A, Ogunyemi O, Leppert JT et al (2008) Flexible ureteroscopy and laser lithotripsy for single intrarenal stones $2 \mathrm{~cm}$ or greater--is this the new frontier. J Urol 179:981-984

[6] Miernik A, Wilhelm K, Ardelt PU et al (2012) Standardized flexible ureteroscopic technique to improve stone-free rates. Urology 80:1198-1202

[7] Joshi HB, Stainthorpe A, MacDonagh RP et al (2003) Indwelling ureteral stents: evaluation of symptoms, quality of life and utility. J Urol 169:1065-1069; discussion 1069

[8] Joshi HB, Stainthorpe A, Keeley FX Jr et al (2001) Indwelling ureteral stents: evaluation of quality of life to aid outcome analysis. J Endourol 15:151-154

[9] Shigemura K, Yasufuku T, Yamanaka K et al (2012) How long should double J stent be kept in after ureteroscopic lithotripsy. Urol Res 40:373-376

[10] Lumma PP, Schneider P, Strauss A et al (2013) Impact of ureteral stenting prior to ureterorenoscopy on stone-free rates and complications. World J Urol 31:855-859

[11] Shields JM, Bird VG, Graves R et al (2009) Impact of preoperative ureteral stenting on outcome of ureteroscopic treatment for urinary lithiasis. J Urol 182:2768-2774

[12] Netsch C, Knipper S, Bach T et al (2012) Impact of preoperative ureteral stenting on stone-free rates of ureteroscopy for nephroureterolithiasis: a matched-paired analysis of 286 patients. Urology 80:1214-1219

[13] Kawahara T, Ito H, Terao H et al (2012) Preoperative stenting for ureteroscopic lithotripsy for a large renal stone. Int J Urol 19:881-885

[14] Rubenstein RA, Zhao LC, Loeb S et al (2007) Prestenting improves ureteroscopic stone-free rates. J Endourol 21:12771280

[15] Ambani SN, Faerber GJ, Roberts WW et al (2013) Ureteral stents for impassable ureteroscopy. J Endourol 27:549-553

[16] Mahajan PM, Padhye AS, Bhave AA et al (2009) Is stenting required before retrograde intrarenal surgery with access sheath. Indian J Urol 25:326-328

[17] Cetti RJ, Biers S, Keoghane SR (2011) The difficult ureter: what is the incidence of pre-stenting. Ann R Coll Surg Engl 93:31-33

[18] Chu L, Sternberg KM, Averch TD (2011) Preoperative stenting decreases operative time and reoperative rates of ureteroscopy. J Endourol 25:751-754

[19] Ito H, Sakamaki K, Kawahara T, Terao H, Yasuda K, Kuroda S, Yao M, Kubota Y and Matsuzaki J. Development and internal validation of a nomogram for predicting stone-free status after flexible ureteroscopy for renal stones. BJU Int 2015; 115: 446-451.

[20] Skolarikos A, Gross AJ, Krebs A et al (2015) Outcomes of Flexible Ureterorenoscopy for Solitary Renal Stones in the CROES URS Global Study. J Urol 
243

244

245

246

247

248

249

250

251

252

253

254

255
[21] Jessen JP, Honeck P, Knoll T et al (2014) Flexible ureterorenoscopy for lower pole stones: influence of the collecting system's anatomy. J Endourol 28:146-151

[22] Resorlu B, Oguz U, Resorlu EB et al (2012) The impact of pelvicaliceal anatomy on the success of retrograde intrarenal surgery in patients with lower pole renal stones. Urology 79:61-66

[23] Perlmutter AE, Talug C, Tarry WF et al (2008) Impact of stone location on success rates of endoscopic lithotripsy for nephrolithiasis. Urology 71:214-217

[24] Hussain M, Acher P, Penev B et al (2011) Redefining the limits of flexible ureterorenoscopy. J Endourol 25:45-49

[25] Shigemura K, Yasufuku T, Yamanaka K et al (2012) How long should double J stent be kept in after ureteroscopic lithotripsy. Urol Res 40:373-376

[26] Traxer O, Thomas A (2013) Prospective evaluation and classification of ureteral wall injuries resulting from insertion of a ureteral access sheath during retrograde intrarenal surgery. J Urol 189:580-584

[27] Assimos D, Crisci A, Culkin D et al (2015) Pre-operative double J stent placement in ureteral and renal stone treatment: results from the Clinical Research Office of Endourological Society URS Global Study. BJU Int 